\title{
Double Inferior Vena Cava with Azygos Continuation and Retroaortic Left Renal Vein Associated with Juxtarenal Abdominal Aortic Aneurysm Surgery
}

\author{
Koji Onoda, MD, PhD, ${ }^{1}$ Yu Shomura, MD, PhD, ${ }^{1}$ and Takuya Komada, MD, PhD ${ }^{2}$
}

The combination of a double inferior vena cava (IVC), a retroaortic left renal vein, and azygos continuation of the IVC is extremely rare. Here we report the case of a 74-year-old man with this complex venous anomaly associated with a juxtarenal abdominal aortic aneurysm (AAA), who underwent a successful graft implantation with left renal artery reconstruction without injuring the retroaortic venous system. The venous anomaly was diagnosed using preoperative contrast-enhanced computed tomography (CT). Thus, contrast-enhanced CT can provide sufficient information on AAA and anomalous venous anatomy to avoid fatal complications during AAA surgery.

Keywords: venous anomaly, retroaortic left renal vein, abdominal aortic aneurysm

\section{Introduction}

Major anomalies of the venous system associated with abdominal aortic aneurysm (AAA), including a retroaortic left renal vein, circumaortic left renal vein, double inferior vena cava (IVC), and left-sided IVC, are unusual. ${ }^{1,2)}$ Careful preoperative assessment and intraoperative recogni-

${ }^{1}$ Department of Cardiovascular Surgery, Shingu Municipal Medical Center, Shingu, Wakayama, Japan

${ }^{2}$ Department of Cardiovascular Surgery, Matsusaka Chuo General Hospital, Matsusaka, Mie, Japan

Received: August 4, 2017; Accepted: October 31, 2017

Corresponding author: Koji Onoda, MD, PhD. Department of Cardiovascular Surgery, Shingu Municipal Medical Center, 18-7

Hachibuse, Shingu, Wakayama 647-0072, Japan

Tel: +81-735-31-3333, Fax: +81-735-31-3335

E-mail: koonoda7832@qc.commufa.jp

(cc) BY-NC-SA (9)2018 The Editorial Committee of Annals of Vascular Diseases. This article is distributed under the terms of the Creative Commons Attribution License, which permits use, distribution, and reproduction in any medium, provided the credit of the original work, a link to the license, and indication of any change are properly given, and the original work is not used for commercial purposes. Remixed or transformed contributions must be distributed under the same license as the original. tion of such anomalies would greatly contribute to reducing or eliminating the risks of severe hemorrhage during abdominal aortic surgery. ${ }^{3,4)}$

Here we present a case of juxtarenal AAA with double IVC, a retroaortic left renal vein, and azygos continuation of the IVC.

\section{Case Report}

A 74-year-old man was admitted to our hospital for the elective repair of an enlarged juxtarenal AAA. He underwent off-pump coronary artery bypass grafting of the left internal thoracic artery to the left anterior descending artery 7 months previously and open cholecystectomy for gallbladder stones 18 years previously. Moreover, he had received radiation therapy for prostatic cancer 3 months prior to the current admission. Physical examination revealed that he was obese (height, $163 \mathrm{~cm}$; weight, $80 \mathrm{~kg}$ ) and his blood pressure was $135 / 70 \mathrm{mmHg}$. Renal function was impaired, with serum creatinine levels of $1.5 \mathrm{mg} / \mathrm{dL}$ and creatinine clearance of $48.9 \mathrm{~mL} / \mathrm{min}$. Contrast-enhanced computed tomography (CT) showed a juxtarenal AAA measuring $50.9 \mathrm{~mm}$ at its maximum diameter, which had increased by $6 \mathrm{~mm}$ in the last 6 months; the aneurysm involved aortic bifurcation and both proximal common iliac arteries. Furthermore, major venous anomalies were revealed-the left renal vein and left IVC met and crossed posterior to the aorta to join the right IVC and continued cephalad as the azygos vein (Figs. 1 and 2C). The azygos vein finally joined the superior vena cava in the thorax (Fig. 2A). The intrahepatic IVC was interrupted (Fig. 2B), and the hepatic segment of the IVC directly drained into the right atrium (Fig. 2D). These findings led to the diagnosis of a juxtarenal AAA accompanied with double IVC, a retroaortic left renal vein, and azygos continuation of the IVC.

The patient was operated on through a vertical midline abdominal incision. After removing the adhesions of the omentum and bowels, the juxtarenal abdominal aorta was exposed, and the taping of bilateral renal arteries was per- 

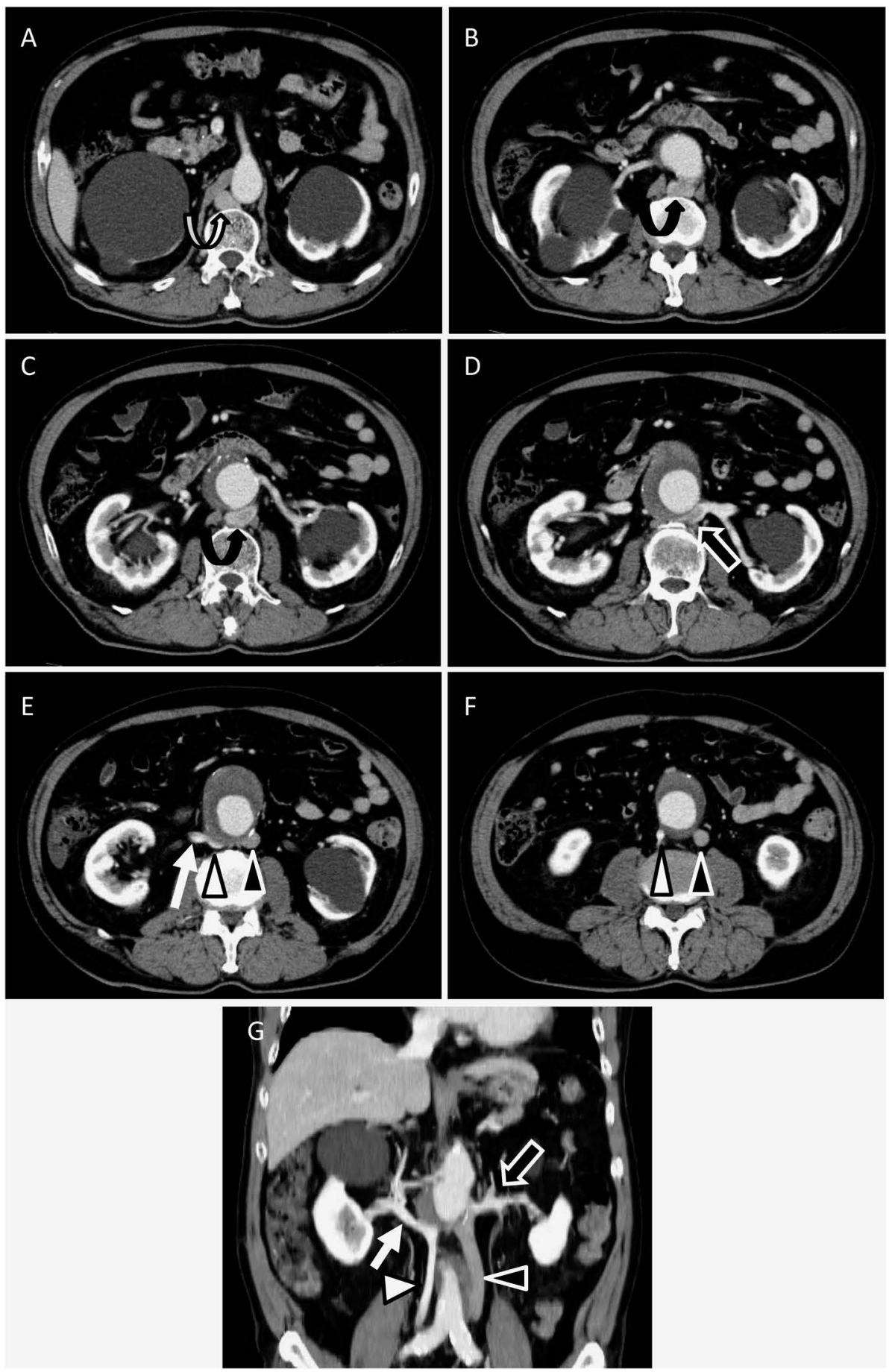

Fig. 1 Preoperative contrast-enhanced axial computed tomography (CT) images from cranial to caudal sections and a coronal image showing a complex venous anomaly and a juxtarenal abdominal aortic aneurysm (AAA). (A) CT obtained at the level of the superior mesenteric artery shows the azygos vein (white curved arrow) within the retrocrural space. (B, C) The juxtarenal AAA is seen at the levels of bilateral renal arteries. The inferior vena cava (IVC) (black curved arrow) running behind the aorta. (D) The left renal vein (black arrow) joins the left IVC and crosses posterior to the aorta. (E) The right renal vein (white arrow) joins the right IVC (white arrowhead). The black arrowhead indicates the left IVC. (F) The double IVC (white and black arrowheads) is observed below renal veins. (G) The coronal CT image shows the double IVC and retroaortic left renal vein (black arrow). The white arrow indicates the right renal vein, and the white and black arrowheads indicate the right and left IVCs, respectively. 

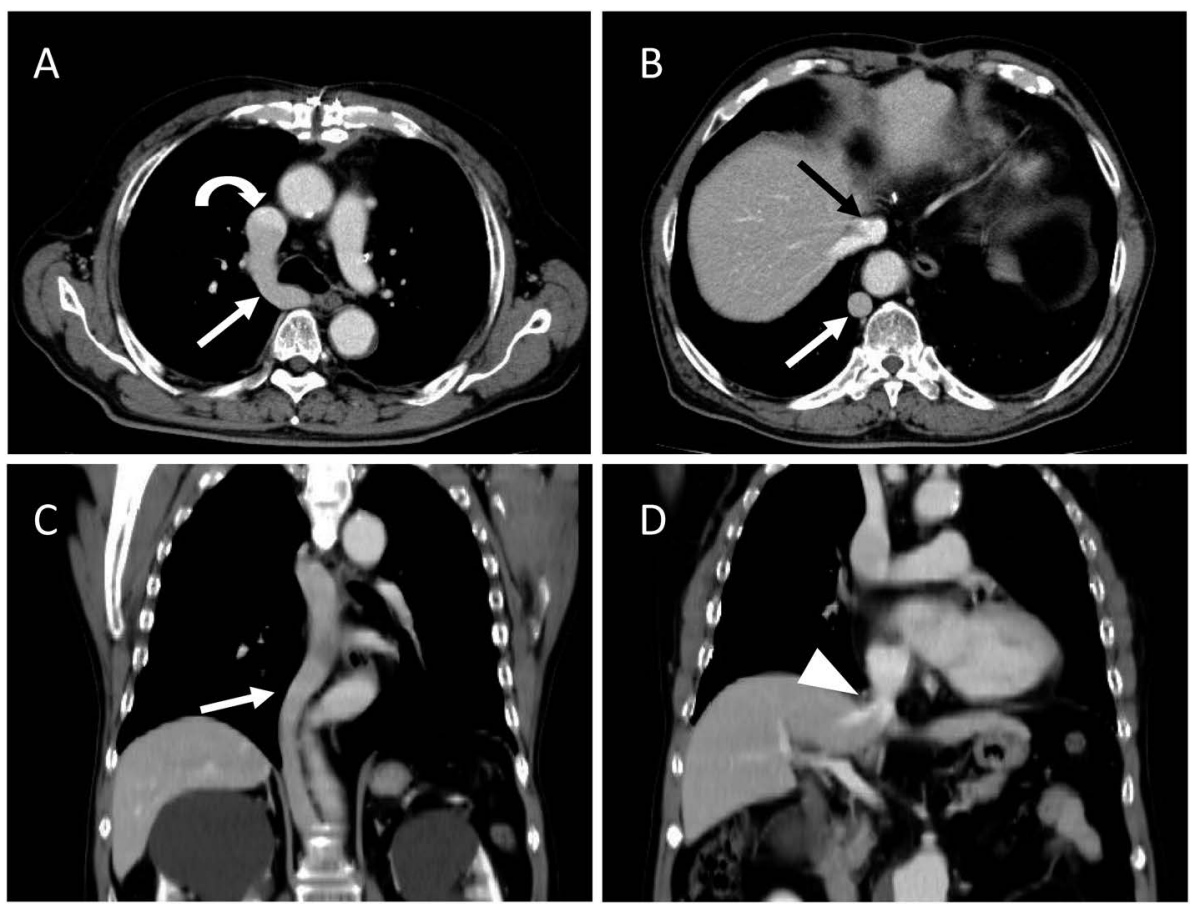

Fig. 2 Preoperative contrast-enhanced computed tomography (CT). (A) CT obtained at the level of the azygos venous arch shows the enlarged azygos vein (straight arrow) draining into the superior vena cava (curved arrow). (B) CT obtained at the level of hepatic veins demonstrates an interrupted intrahepatic IVC (black arrow) and azygos vein (white arrow). (C) Coronal CT image shows the enlarged azygos vein (straight arrow). (D) Coronal CT image shows that the hepatic segment of the IVC (white arrowhead) directly drains into the right atrium.

formed using the Omni retractor system. After the systemic administration of heparin, the suprarenal abdominal aorta, bilateral renal arteries, and bilateral common iliac arteries were clamped. Particularly, the aorta was carefully clamped to prevent injury to the azygos vein within the retrocrural space. Once the aorta was incised just below the left renal artery with care to prevent injuring the IVC behind the aorta, the retroaortic left renal vein was caudally exposed. Additional dissection was performed cephalad. Subsequently, a $20-\times 10-\mathrm{mm}$ bifurcated vascular prosthesis (J Graft SHIELD NEO, JUNKEN MEDICAL Co., Ltd., Tokyo, Japan) was implanted just below the right renal artery. The left renal artery was reconstructed in the left limb of the graft using a 7-mm vascular prosthesis (J Graft SHIELD NEO, JUNKEN MEDICAL Co., Ltd., Tokyo, Japan). Renal protection was achieved by infusing $250 \mathrm{~mL}$ of iced Ringer's solution into each renal artery. The cross-clamp time of right and left renal arteries was 85 and $105 \mathrm{~min}$, respectively, and the total blood loss was $860 \mathrm{~mL}$. The patient had an uneventful postoperative course, except for deteriorated renal function. Although his serum creatinine level was $3.69 \mathrm{mg} / \mathrm{dL}$ on postoperative day 1 , it gradually ameliorated to $2.00 \mathrm{mg} / \mathrm{dL}$, without temporary dialysis, on postoperative day 15. At the 12-month follow-up, the patient was doing well, with a serum creatinine level of $1.67 \mathrm{mg} / \mathrm{dL}$.

\section{Discussion}

Normal IVC comprises four segments, namely hepatic, suprarenal, renal, and infrarenal. The infrahepatic IVC develops between 6 and 8 weeks of embryonic life. The embryonic development of the IVC is a complex process that includes the formation, regression, and fusion of three pairs of primitive veins, namely posterior cardinal, subcardinal, and supracardinal veins. ${ }^{3,5}$ )

As reported by Bass et al., major anomalies of the venous system are double (prevalence, $0.2 \%-3 \%$ ) and left $(0.2 \%-0.5 \%)$ IVC, retroaortic $(2.1 \%)$ and circumaortic $(8.7 \%)$ left renal vein, and the absence of the hepatic segment of the IVC with azygos continuation $(0.6 \%) .^{5)}$ The combination of a double IVC, a retroaortic left renal vein, and azygos continuation of the IVC is extremely rare. ${ }^{3)}$ This embryonic event is theorized to occur because of the persistence of the left supracardinal vein and dorsal limb of the renal collar with regression of the ventral limb. In addition, when the subcardinal-hepatic anastomosis fails to develop, the hepatic segment of the IVC independently drains directly into the right atrium. ${ }^{5)}$

The recognition of this venous anomaly is important in procedures such as AAA surgery, venous thromboembolism, IVC filter placement, temporary pacing through 
the transfemoral route, cardiopulmonary bypass surgery, and palliative systemic venous-pulmonary artery shunt surgery. ${ }^{3)}$

Two widely adapted approaches for the open aneurysmal repair of a juxtarenal AAA are transperitoneal and retroperitoneal. Unlike in the transperitoneal approach, the obscuring left renal vein and body of the pancreas are not limiting factors in the retroperitoneal approach. Therefore, the latter approach is ideal for juxtarenal or suprarenal aortic exposure. $\left.{ }^{6}\right)$ The retroperitoneal approach is also recommended for cases of an infrarenal AAA with a retroaortic left renal vein because keeping the left kidney down in its bed rather than anteriorly mobilizing it may facilitate the exposure of the aneurysmal neck, thereby minimizing the risks of venous injury. ${ }^{7)}$ However, there are few advantages to keeping the left kidney down during suprarenal aortic exposure while using the retroperitoneal approach. Moreover, a relative contraindication to this approach is the presence of a double or left IVC. ${ }^{7}$ ) Therefore, we chose the transperitoneal approach in our case although the patient previously underwent an open cholecystectomy.

The presence of a venous anomaly may cause massive bleeding during AAA surgery. Specifically, some authors have reported unexpected bleeding from venous injuries during the dissection of the abdominal aorta at the proximal anastomotic site..$^{4,8-10)}$ Such reports have emphasized the importance of the careful identification of a retroaortic left renal vein before aortic surgery to avoid severe hemorrhage. In our case, special care was taken to prevent injuring the IVC behind the aorta and azygos vein within the retrocrural space during the dissection of the aneurysmal neck or placement of the proximal aortic clamp. By incising the aorta just below the left renal artery, the left renal vein running behind the aorta was caudally identified, and the graft was then implanted without injuring the retroaortic venous system. Thus, preoperative contrastenhanced CT was useful for both evaluating the AAA and confirming the presence of venous anomaly.

In our case, CT images were obtained using an 80-row MDCT scanner (Aquilion ${ }^{\mathrm{TM}}$ PRIME, Toshiba Medical System Co., Ohtawara, Tochigi, Japan). A total of $100 \mathrm{~mL}$ of contrast medium was injected at a rate of $3.3 \mathrm{~mL} / \mathrm{s}$. The scan delay from the beginning of contrast injection to the venous phases was $90 \mathrm{~s}$. Because little contrast material was present in the left IVC (Fig. 1), a longer scan delay was needed to obtain clearer images of venous structures. Therefore, we have displayed axial and coronal CT images of anomalous venous structures instead of three-dimensional CT images. Conversely, in cases of AAA surgery, venous structures may be imaged only during the arterial phase; consequently, the veins would contain little or no contrast medium during preoperative contrast-enhanced CT. Hence, familiarity with anomalous venous structures is essential for an accurate interpretation of cross-sectional images obtained during the arterial phase.

\section{Conclusion}

We reported a case of a double IVC with a retroaortic left renal vein and azygos continuation of the IVC associated with a juxtarenal AAA. Our results indicate that preoperative contrast-enhanced CT can provide details of AAA and anomalous venous anatomy in patients undergoing AAA repair. The precise recognition of such anatomies is important to avoid fatal complications during surgery.

\section{Disclosure Statement}

All authors have no conflict of interest to declare.

\section{Author Contributions}

\section{Writing: $\mathrm{KO}$}

Critical review and revision: all authors

Final approval of the article: all authors

Accountability for all aspects of the work: all authors

\section{References}

1) Bartle EJ, Pearce WH, Sun JH, et al. Infrarenal venous anomalies and aortic surgery: avoiding vascular injury. J Vasc Surg 1987; 6: 590-3.

2) Baldridge ED Jr, Canos AJ. Venous anomalies encountered in aortoiliac surgery. Arch Surg 1987; 122: 1184-8.

3) Ahmetoglu A, Cansu A. Duplication of the inferior vena cava with azygos continuation, retroaortic left renal vein and iliac vein variations. Bratisl Lek Listy 2012; 113: 448-50.

4) Shindo S, Kubota K, Kojima A, et al. Anomalies of inferior vena cava and left renal vein: risks in aortic surgery. Ann Vasc Surg 2000; 14: 393-6.

5) Bass JE, Redwine MD, Kramer LA, et al. Spectrum of congenital anomalies of the inferior vena cava: cross-sectional imaging findings. Radiographics 2000; 20: 639-52.

6) Ernst CB. Retroperitoneal exposure of abdominal aorta. In: Haimovici H, Ascer E, Hollier LH, et al. eds. Haimovici’s Vascular Surgery, 4th edition. Massachusetts: Blackwell Science, 1996: 393-7.

7) Karkos CD, Bruce IA, Thomson GJL, et al. Retroaortic left renal vein and its implications in abdominal aortic surgery. Ann Vasc Surg 2001; 15: 703-8.

8) Nonami Y, Yamasaki M, Sato K, et al. Two types of major venous anomalies associated with abdominal aneurysmectomy: a report of two cases. Surgery Today 1996; 26: 940-4.

9) Toda R, Iguro Y, Moriyama Y, et al. Double left renal vein associated with abdominal aortic aneurysm. Ann Thorac Cardiovasc Surg 2001; 7: 113-5.

10) Hashizume K, Taniguchi S, Ariyoshi T, et al. Circumaortic left renal vein associated with juxtarenal abdominal aortic aneurysm. Ann Vasc Dis 2013; 6: 658-61. 